University for Business and Technology in Kosovo

UBT Knowledge Center

UBT International Conference

2017 UBT International Conference

Oct 27th, 1:00 PM - 2:30 PM

\title{
Development trends in Robotics
}

Peter Kopacek

Technische Universität Wien, kopacek@ihrt.tuwien.ac.at

Follow this and additional works at: https://knowledgecenter.ubt-uni.net/conference

Part of the Robotics Commons

\section{Recommended Citation}

Kopacek, Peter, "Development trends in Robotics" (2017). UBT International Conference. 280.

https://knowledgecenter.ubt-uni.net/conference/2017/all-events/280

This Event is brought to you for free and open access by the Publication and Journals at UBT Knowledge Center. It has been accepted for inclusion in UBT International Conference by an authorized administrator of UBT Knowledge Center. For more information, please contact knowledge.center@ubt-uni.net. 


\title{
Development trends in Robotics
}

\author{
P.Kopacek \\ TU Wien, Institute of Mechanics and Mechatronics - IHRT \\ Wiedner Hauptstrasse 9, E325A6 \\ A-1040 Wien \\ kopacek@ihrt.tuwien.ac.at
}

\begin{abstract}
After a short introduction the basic ideas of production 4.0 will be presented. Production 4.0 is not new. We had it under the headline "Agile Manufacturing" in the late Nineties. The idea beyond was to produce different products on one line economically.

The role of robots in production 4.0 will be shortly outlined. The main goal of this contribution is to make some additional remarks to the existing kinds of robots and introduce some new categories probably necessary in the future for agile manufacturing.
\end{abstract}

Keywords: Production Automation, Agile Manufacturing, Robots.

\section{Introduction}

Robots are always a frequently used as an example for Mechatronic Systems. Currently the field of robotics is in the stage of very fast development not only because of the latest trends in production automation.

The last step Production 4.0 is not new. We had it under the headline "Agile Manufacturing" in the late Nineties. The idea beyond was to produce different products on one line economically. At that time it was not realizable because of the missing IT hard- and software.

Main ideas are

- Products and machines will be equipped with processors, sensors and wireless communication facilities

- Communicate with each other and with other production machines, and are selforganizing as well as partially even self-optimizing. .

- Information through data glasses: The emergence of "Gray-collar workers": Channeling of "data cemeteries"

One of the main goals of production 4.0 is to create "Smart Factories". They beginning to appear and employ a completely new approach to production. Smart Factories allow individual customer requirements and the dynamic business and engineering processes enable last-minute changes to production.

These smart products are

- uniquely identifiable,

- may be located at all times

- know their own history and the current status

- able to find alternative routes to achieving their target (final) state 


\section{Development trends}

In manufacturing automation Industry 4.0 is the next phase in the digitalization of the manufacturing sector.

It is sometimes called the $4^{\text {th }}$ industrial revolution.

It is driven by:

Rise in data volumes

Computational power

Connectivity

Virtual to real world data transformation

Not only for this one of the most ambitious aims of robotics is to design autonomous robots (industrial, mobile, humanoid) that could reach - and even surpass - human intelligence and performance in partially unknown, changing, and unpredictable environments.

This goal is closely connected with the developments of Artificial Intelligence (AI). AI will be able to lead the robot to fulfill the missions required by the end users. To achieve this goal, over the past decades scientists have worked on AI techniques in many fields, including:

1. Artificial intelligence

2. Perception and analysis of the environment

3. Natural language processing

4. Human interaction

5. Cognitive systems

6. Machine learning and behaviors

7. Neural networks

Therefore in the overview (Fig.1) conventional as well as "advanced" robots fullfill total or partially some of the features mentioned above are tried to systemize. 


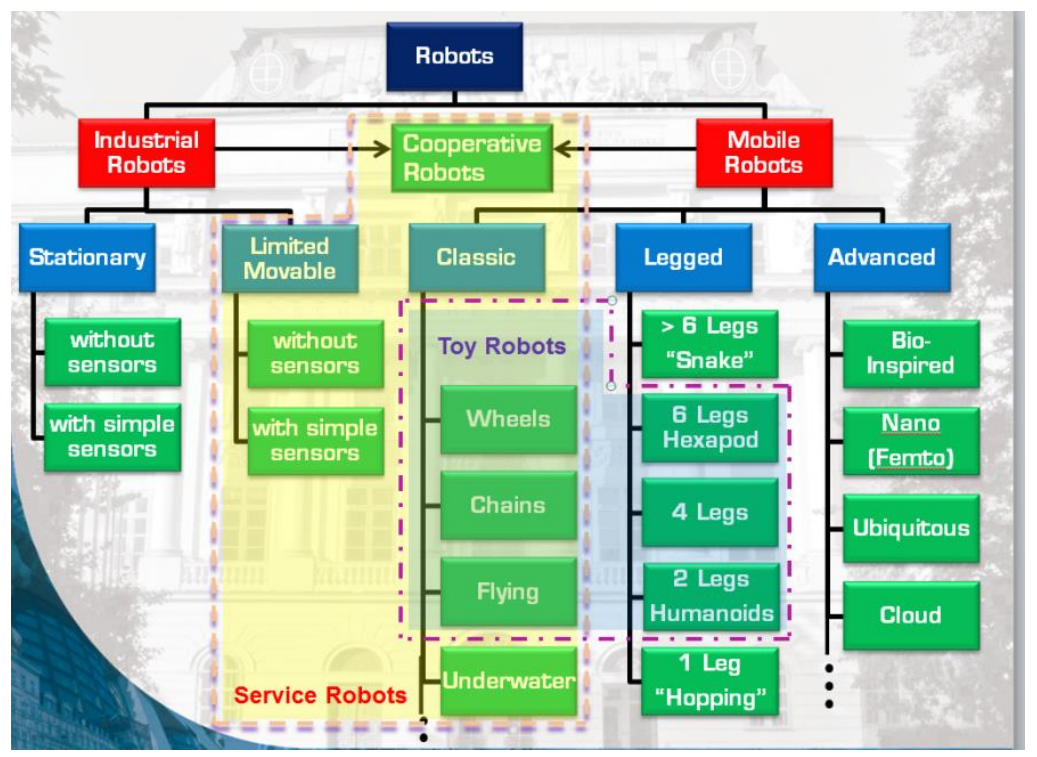

Fig.1 Robots

Clearly this new automation philosophy requires highly cooperative industrial, mobile and probably in the future humanoid robots - a so called multi-robot system.

A multi-robot system is a distributed system that consists of a collection of autonomous computers, connected through a network and distribution middleware which enables them to coordinate their activities and to share the resources of the system so that user perceives the system as a single, integrated computing facility. Nevertheless, multiple robot systems are different from any other distributed systems because of their implicit "real world" environment, which is more difficult to model.

A system involving several robots is a distributed system as distribution exists in a spatial and informative way as well as regarding decision making. Spatial distribution exists as each robot is located in a different position. Besides, informative distribution is clear as information belongs to each robot so that not all information is common to all agents. At the end, the last decision about the task is always inside each robot that would execute the tasks depending on its intern situation.

The term collective behavior denotes any behavior of agents in a system having more than one agent. Therefore, cooperative behavior is a subclass of collective behavior characterized by cooperation, this is, the ability to work or act together for a common purpose.

Hence it follows that a multi- robot system displays cooperative behavior if due to some underlying mechanism, there is an increase in the total utility of the system. There are three fundamental aspects for cooperative behavior: the task the robots must perform, the mechanism of cooperation and the system performance

In this context, one of the fundamental aspects of the robots is their capability to learn: to learn the characteristics of the surrounding environment, that is, the physical environment, but also the living beings that inhabit in. This means that robots working in a given environment have to distinguish human beings from other objects. 
In addition to learning about their environment, robots have to learn about their own behavior, through a self-reflective process. They have to learn from experience, replicating somehow the natural processes of the evolution of intelligence in living beings (synthesis procedures, trying and error, learning by doing, and so on).

21 th century robots will be used in all areas of modern life. The major challenges are:

- To develop robotic systems that can sense and interact useful with the humans.

- To design robotic systems able to perform complex tasks with a high degree of autonomy.

The role of these robots of the future could be improved by embedding them into emerging IT environments characterized by a growing spread of ubiquitous and cloud computing, communications and of ad-hoc networks of sensors forming what has been termed "ambient intelligence".

\section{Selected Examples}

In the following some examples are described and shortly discussed which will be used in the very near future in industry or in daily life of humans.

\section{Dual arm robots}

Dual arm industrial robots are known since more than 5 years. They have usually only an upper body with two arms and a gripping unit. Because of their $60-70$ degrees of freedom (DOF`s) industrial applications are for complex handling and assembly tasks.

One "cost oriented" example is Baxter an industrial android (humanoid robot) that can work right next to line employees on the factory floor, often working on highly repetitive tasks such as precision packing.

- Learns easily: No teach box is needed. Nontechnical users can physically guide Baxter through the motions that they want it to perform.

- Versatility: Can perform a wide range of simple tasks.

- No programming required: No need to hire third-party application developers.

- Adaptability: Can adapt automatically to changes in the work environment.

- Safety: Users do not need to deploy protective cages.

- Interchangeable end effectors: Choice of tools that can be easily swapped at the end of either arm.

\section{Cooperative robots}

Robots can accomplish different tasks in different environments, tasks that are tedious, difficult or even impossible for a human operator. If several robots are combined in order to create a multirobot system, the range of tasks they can perform increases even more, as these systems can carry out actions that no single robot could since they are always spatially limited, no matter how capable they are. 
A multi-robot system is a distributed system that consists of a collection of autonomous robots, connected through a network and distribution middleware which enables them to coordinate their activities and to share the resources of the system so that user perceives the system as a single, integrated computing facility. Nevertheless, multiple robot systems are different from any other distributed systems because of their implicit "real world" environment, which is more difficult to model.

\section{Collaborative robots - COBOTS}

In order to perform collaborative tasks Cobots are designed to be safe around humans (using sensors, force limiting and rounder geometries than traditional robots), to be lightweight (for them to be moved from task to task) and to be easy to implement and use without skills in programming. Above all, a collaborative robot is not a replacement robot; it assists workers rather than replaces them.

As detailed by the international ISO10218 standard, robots can have four types of collaborative features. These are Safety Monitored Stop, Hand Guiding, Speed and Separation Monitoring and Power and Force Limiting.

Safety Monitored Stop is implemented in environments where the robots operate mostly alone, with occasional human interference. The feature will cause the robot to pause (though not shutdown) when the safety zone is violated (i.e. a human enters its workspace).

The speed and separation monitoring feature is an extension of Safety Monitored Stop. Instead of adopting a single behavior throughout the robot's entire workspace, the latter is gradated into several safety zones.

Hand Guiding enables the robot to move while the worker is in its workspace (as is possible with Speed and Separation Monitoring). Using an end-of-arm device capable of detecting applied forces, the robot can be guided by a worker for hand guiding and rapid path teaching.

Power and Force Limiting feature is probably the safest of them all as these type of robots are purposefully designed to operate around humans rather than adapted to do so. As the name suggests, the forces and energy applied to a human are limited so as to avoid any inflicted harm.

\section{Driverless mobile robots}

Delivery robots are mobile robots that are capable of locomotion. „they have the ability to move around the environment and are not fixed to one physical location. Mobile robots can be "autonomous" (AMR - autonomous mobile robot) which means they are capable of navigating an uncontrolled environment without the need for physical or electro-mechanical guidance devices. Alternatively, mobile robots can rely on guidance devices that allow them to travel a pre-defined navigation route in relatively controlled space (AGV) - autonomous guided vehicle). By contrast, industrial robots are usually more-or-less stationary, consisting of a jointed arm (multi-linked manipulator) and gripper assembly (or end effector), attached to a fixed surface."

\section{Flying Robots}

An unmanned aerial vehicle (UAV), especially drones are aircrafts without a human pilot aboard. Besides these two names they are RPAS (remote piloted aircraft systems) and model aircraft. The term "drone" seems to be more common for military use whereas "UAV" is more common for hobby use. 
Drones are controlled by autonomously onboard computers or by from a remote control of a pilot on the ground or in another vehicle. They can carry a lethal or nonlethal payload.

The purpose of this device is to replace the human and to do the uncomfortable, risky or unpleasant jobs Drones are often preferred for missions that are too "dirty or dangerous" for manned aircraft.

\section{Snake Robots}

Snake robots, also known as hyper redundant mechanisms, use their many degrees of freedom to achieve locomotion. They vary a lot in their form and dimensions. They can be four stories long or small enough to move around the organs inside humans. Some important characteristics of snake-bots are their small cross section to

length ratio which allows them to move through tight spaces and second being their ability to transform their body shape to perform different behaviors such as climbing staircases or trees.

Snake robots are manufactured by chaining a number of individual links. This trait helps them to avoid failure and emulates their real life counterpart as the snake-bot can continue to function even though some parts of its body are ruined.

Their ability to maneuver a variety of terrain types, redundancy and also their ability to complete seal of their

bodies are some of their main features. Snake-bots unique characteristics give them an advantage in maneuvering the environment. Snake-bots are in development to assist in search and rescue operations. Whenever there are a number of hindrances and obstructions in the environment that need to be overcome, the locomotive flexibility of snake-bots make them highly reliable for such a scenario.

\section{Summary and outlook}

After a short introduction the necessary basic ideas of production 4.0 are presented. The role of robots in form of cooperative robots (Multi- agent systems) is shortly outlined. One of the main goals of production 4.0 is to create "Smart Factories". They beginning to appear and employ a completely new approach to production. Smart Factories allow individual customer requirements and the dynamic business and engineering processes enable last-minute changes to production.

Clearly this new automation philosophy requires highly cooperative industrial, mobile and probably in the future humanoid robots - a so called multi-robot system. For this cooperative and collaborative robots are necessary. These are shortly described as well as some others used or probably will be used in the future not only in agile manufacturing.

Open questions are social and ethical aspects of these production philosophies.

\section{Reference}

7. Kopacek, P. (2017): Unpublished lecture material. TU Wien/IHRT 